\title{
Impact of Shuffle on Trajectories on Certain Classes of Partial array Languages
}

\author{
H. Geetha ${ }^{1}$ K. Suresh ${ }^{2}$ P. Venkatesh Babu ${ }^{3}$ and T. Kalyani ${ }^{4}$ \\ ${ }_{1,2,4}$ Department of Mathematics, \\ 1,2 St. Joseph's College of Engineering, \\ ${ }^{4}$ St. Joseph's Institute of Technology \\ Chennai-119, Tamilnadu, India. \\ ${ }^{3}$ Department of Architecture, \\ SRM SEAD, SRMIST, \\ Chennai-89, Tamil Nadu, India. \\ E-mail: geethahvenki@hotmail.com,dhivasuresh@gmail.com,sp.v.babu@gmail.com
}

\begin{abstract}
In the recent years many tools are being used for developing picture languages. The vital and most interesting among such tools is the shuffle on trajectories. The above tool can be applied on various disciplines. In this paper,a Finite State Matrix Partial array Automaton is defined and someresults over Finite State Matrix Partial array Automaton and Online Tessellation Partial Automaton are studied.
\end{abstract}

Key Words: Finite State Matrix Partial Array automaton, online Tessellation Partial Automaton

\section{Introduction}

Berstel and Boasson [1] introduced the concept of partial words in their study on biological molecules. This concept of partial words was converted to arrays and thus the partial arrays were introduced and their combinatorial studies were also done. The recognizability of partial arrays using online tessellation automaton was studied in depth in [7]. The introduction of shuffle on trajectories and syntactic constraints were studied by mateescu et.al [5]. The concept of trajectories was applied to contextual array grammars in [4]. The shuffle on trajectories over finite array languages was studied [2]. Shuffle on array languages generated by array grammars was discussed in [6]. Inspired by the above studies, the tool shuffle on trajectories over partial array languages and its effects on certain classes of partial arrays were introduced [3].

In this paper we define Finite State Matrix Partial Array automaton and applied shuffle on trajectories to arrive two interesting theorems.

\section{Preliminaries}

In this section we give the definition of partial array and online Tessellation partial automaton.

\section{Definition. 1}

A deterministic online tessellation partial automaton (OTPA) is $\mathrm{B}=\left(\Sigma \cup\{\oslash\}, Q, Q_{h}, q_{0}, F, \delta\right)$ where $\mathrm{Q}$ is a set of states, $Q_{h}$ is the states associated with the holes $\diamond . q_{0} \in Q$ is the begining state. $F \subseteq\left(Q \cup Q_{h}\right)$ is a set of finishing states, the mapping $\delta$ function defined as $\delta: Q_{1} \times Q_{1} \times \Sigma \cup\{\oslash\} \rightarrow Q_{1}$. Where $Q_{1}=Q \cup Q_{h}$. For computation of a two-dimensional OTPA we refer [7]. The language of finite partial arrays recognized by OTPA, B is denoted by $L(\mathrm{~B})$ and $\mathrm{L}(O T P A)$ is the set of partial array languages recognized by OTPA.

\section{Main Results}

In this section we define finite state matrix partial array automaton and a theorem on regular partial matrix array languages and on recognizable partial array Languages.

\section{Definition. 2}

A finite state matrix partial array automaton (FSMPA)is constructedas B $=\left(Q_{h}, \Sigma \cup\{\oslash\}, \Gamma, \delta, \delta, S_{h}, F_{h}, F_{h}^{\prime}, \$\right)$ where $Q_{h}=\bar{Q}_{h} \cup Q_{1 h} \cup \ldots \cup Q_{k h}, Q_{i h} \cap Q_{j h}=\phi_{1}$ for $i \neq j$, that includes the states associated to $\diamond . \Sigma$ and $\Gamma$ are the finite set of inputs and stack symbols respectively, with $\#|\Gamma|=k$. Each $Q_{i h}$ has an initial state either $\left\{q_{i}\right\}$ or 
$\left\{q_{h i}\right\}$ and a final state $\left\{f_{i}\right\}$ or $\left\{f_{h i}\right\} i=1, \cdots, k$. Define $\left.F_{h}^{\prime}=\bigcup_{i=1}^{k}\left(\left\{f_{i}\right\} \cup\left\{f_{h i}\right\}\right\}\right)$ is the set of transition states. $S_{h}=\bigcup_{i=1}^{k}\left(\left\{q_{i}\right\} \cup\left\{q_{h i}\right\}\right)$ the set of start states. $\bar{Q}_{h}$ has an initial state $q_{0}$ and $F_{h} \subseteq \bar{Q}_{h}$ is the set of end states. $\$ \not \Sigma$ is to denote the end $\delta$ is defined as $\delta: Q_{i h} \times \Sigma \cup\{\emptyset\} \rightarrow Q_{i h} \times\{\varepsilon\},\left\{f_{i}\right\} \cup\left\{f_{h i}\right\} \times \$$ into $\left(S_{h} \cup\left\{q_{0}\right\}\right) \times s_{i}$, here $S_{i}$ is the stack withrespect to $Q_{i h}$. $\delta^{\prime}$ is defined from $\bar{Q}_{h} \times \Gamma$ into the finite subsets of $\bar{Q}_{h}$. Initially, the input matrix is placed at the end marker.

where $b_{i j} \in \Sigma \cup\{\otimes\}, 1 \leq i \leq m$ and $1 \leq j \leq n$.

$$
\begin{array}{ccc}
\$ & \ldots & \$ \\
b_{m 1} & \ldots & b_{m n} \\
\ldots & \ldots & \ldots \\
b_{i 1} & \ldots & b_{i n}
\end{array}
$$

To begin with, the FSMPA starts recognizing from bottom to top as per $\delta$ transition, after reaching the first $\$$ it writes a symbol from $\Gamma$ and then reaches the next column. This process is repeated untill the matrix of size $m \times n$ is read. At this stage the storage will have $n$ symbols. Now, using $\delta^{\prime}$ from left to right and attains final state, if not the matrix is rejected.

The configuration $(p,(i, j), x, t)$ where $p$ the present state in $Q_{h},(i, j)$ is the location of the input, $x$ is a partial word, $t$ is the number of positions from left.

If $(q,(m+1, n), x, r)$ is an arrangement and $\delta^{\prime}(q, z)$ has $q^{\prime}$ implies

$(q,(m+1, n), x, r) \underset{\delta^{\prime}}{\square}\left(q^{\prime},(m+1, n), z, r+1\right)$ where $q$ belongs to $F_{h}^{\prime}$ and $q^{\prime}$ is in $S_{h}$.

\section{Definition. 3}

The set of all languages accepted by FSMPA is denoted as

$L(\mathrm{~B})=\left\{\left[b_{i j}\right], i=1,2, \cdots m, j=1,2, \cdots n, m, n \geq 1 / b_{i j} \in \Sigma \cup\{\oslash\}\right.$,

$(p,(1,1), \varepsilon, 1) \underset{\delta}{\square_{\delta}^{*}}\left(q_{0},(m+1, n), x, 1\right) \underset{\delta^{\prime}}{\square^{*}}\left(p^{\prime},(m+1, n), x, n\right)$

with $p$ in $S_{h}, p^{\prime}$ in $F_{h}$ and $x$ in $\left.\Gamma^{+}\right\}$

Note: The languages accepted by finite state matrix partial array automaton (FSMPA) are all regular partial matrix array languages.

\section{Theorem. 1}

$L_{1}$ and $L_{2}$ are the regular partial matrix array languages and if $T \subseteq\{r, u\}^{*} \cup\{l, d\}^{*}$, a regular set of trajectories, then $L_{1} \amalg_{\mathrm{T}} L_{2}$ is also a regular partial matrix array language.

\section{Proof.}

Let $\mathrm{A}_{1}=\left(Q_{1 h}, \Sigma \cup\{\diamond\}, \Gamma_{1}, \delta_{1}, \delta_{1}^{\prime}, S_{1 h}, F_{1 h}, F_{1 h}^{\prime}, \$\right)$ and

$\mathrm{A}_{2}=\left(Q_{2 h}, \Sigma \cup\{0\}, \Gamma_{2}, \delta_{2}, \delta_{2}^{\prime}, S_{2 h}, F_{2 h}, F_{2 h}^{\prime}, \$\right)$ are the two FSMPA and thus $L\left(\mathrm{~A}_{1}\right)=L_{1}$ and $L\left(\mathrm{~A}_{2}\right)=L_{2}$. $Q_{1 h}$ and $Q_{2 h}$ are defined respectively as

$Q_{1 h}=\bar{Q}_{1 h} \cup Q_{11 h} \cup Q_{12 h}, \cdots, \cup Q_{1 k_{h}}$, with $Q_{1 i} \neq Q_{1 j}$ and

$Q_{2 h}=\bar{Q}_{2 h} \cup Q_{21 h} \cup Q_{22 h}, \cdots, \cup Q_{2 k h}$ with $Q_{2 i} \neq Q_{2 j}$ for $i \neq j$,

$\Gamma_{1}$ and $\Gamma_{2}$ are the stack symbols and they correspond to one and only one $Q_{1 i h}$ and $Q_{2 i h}$ respectively. $\left|\Gamma_{1}\right|$ and $\left|\Gamma_{2}\right|$ each equal to $k$. Each $Q_{1 i h}$ and $Q_{2 i h}$ has astart state $\left\{q_{1 i}\right\} \operatorname{or}\left\{q_{h l i}\right\}$ and $q_{2 i}$ or $q_{h 2 i}$. Each with final states $f_{1 i}$ or $f_{h 1 i}$ and $f_{2 i}$ or $f_{h 2 i}$ where $i=1,2, \cdots, k . S_{1 h}=\bigcup_{i=1}^{k}\left\{q_{1 i} \cup q_{h 1 i}\right\}$ and $S_{2 h}=\bigcup_{i=1}^{k}\left\{q_{2 i} \cup q_{h 2 i}\right\}$ are the set of begining states. $F_{1 h}^{\prime}=\bigcup_{i=1}^{k}\left\{f_{1 i} \cup f_{h l i}\right\}$ and $F_{2 h}^{\prime}=\bigcup_{i=1}^{k}\left\{f_{2 i} \cup f_{h 2 i}\right\} . \bar{Q}_{1 h}$ and $\bar{Q}_{2 h}$ have starting states $\left\{q_{10} \cup q_{h 10}\right\}$ and $\left\{q_{20} \cup q_{h 20}\right\}$ respectively. $F_{1 h} \subseteq \bar{Q}_{1 h}, F_{2 h} \subseteq \bar{Q}_{2 h}$ are the end states. $\delta_{1}$ is from $Q_{1 i h} \times \Sigma \cup\{\diamond\}$ into the finite subsets of $Q_{1 i h} \times\{\dot{O}\}, i=1,2, \cdots, k$ and from $f_{1 i} \times \$$ into $\left(S_{1 h} \cup\left\{q_{10} \cup q_{h 10}\right\}\right) \times s_{1 i}$ where $s_{1 i}$ is the stack 
symbol corresponding to $Q_{1 i} . \delta_{1}^{\prime}$ is the mapping from $\bar{Q}_{1 h} \times \Gamma$ into finite subset of $Q_{1 h}$. Similarly we define $\delta_{2}$ and $\delta_{2}^{\prime}$.

Define $\mathrm{A}_{T}=\left(\{r, u, \ell, d\}, q_{0}^{T}, \delta_{T}, q, F_{T}\right)$ is a finite deterministic automaton such that $L\left(\mathrm{~A}_{T}\right)=T$ and the FSMPA, A $=\left(\Sigma \cup\{\diamond\}, \Gamma, Q_{h}, \delta, \delta^{\prime}, S_{h}, F_{h}, F_{h}, \$\right)$ such that $L(\mathrm{~A})=L_{1} \amalg_{\mathrm{T}} L_{2}$. Here $Q_{h}$ is the set of states associated with $\diamond, Q_{h}=\bar{R}_{h} \cup R_{1 h} \cup \ldots \cup R_{k h}, R_{i h} \neq R_{j h}, i \neq j$. Each $R_{i h}$ has astarting state $q_{i}$ or $q h_{i}$ and a final state $f_{i}$ or $f_{h i}, i=1,2, \cdots, k . S_{h}=\bigcup_{i=1}^{k}\left\{q_{i} \cup q_{h i}\right\}, \bar{R}_{h}$ has a start state $q_{0} . F_{h} \subseteq \bar{R}$ is the end state. $\$$ is the end marker. Define $Q_{h}=Q_{1 h} \times Q_{T} \times Q_{2 h}, S_{h}=\left\{\left(q_{0}^{1}, q_{0}^{T}, q_{0}^{2}\right)\right\}$, or more precisely we define $S_{h}$ as $\left\{q_{h 10}\left(q_{h 10}\right), q_{0}^{T}, q_{20}\left(q_{h 20}\right)\right\}$, $F_{h}=F_{1 h} \times F_{T} \times F_{2 h}$ and $F_{h}^{\prime}=F_{1 h}^{\prime} \times F_{T}^{\prime} \times F_{2 h}^{\prime} \mathrm{A}$ run of $\mathrm{A}$ on an input $\mathrm{P} \in(\Sigma \cup\{\otimes\})^{* *}$ imitate either $\mathrm{A}_{1}$ or $\mathrm{A}_{2}$ and as time varies, changes the functioning from $A_{1}$ to $A_{2}$ or from $A_{2}$ to $A_{1}$. For each of the changes the transition in $\mathrm{A}_{T}$ as follows:

If change is from $\mathrm{A}_{1}$ to $\mathrm{A}_{2}$ then it is interpreted as $u$ or $d$ andit is $r$ or $\ell$ respectively if it is from $\mathrm{A}_{2}$ to $\mathrm{A}_{1}$. The input $\mathrm{P}$ is accepted by $\mathrm{A}$ iff $L_{1}, L_{2}$ and $T$ are recongnized byeach of $\mathrm{A}_{1}, \mathrm{~A}_{2}$ and $\mathrm{A}_{T}$ respectively.

The mapping $\delta$ is defined as:

If $\mathrm{T}$ is a column shuffle, then the transition is

$$
\begin{aligned}
\delta\left(\left(q_{1}, q_{T}, q_{2}\right), a\right)= & \left\{\left[\left(\delta_{1}\left(q_{1}, a\right), \delta_{T}\left(q_{T}, r\right), q_{2}\right)\right],\left[\left(\delta_{1}\left(q_{1}, \diamond\right), \delta_{T}\left(q_{T}, r\right), q_{2}\right)\right],\right. \\
& {\left[\left(\delta_{1}\left(q_{1}, a\right), \delta_{T}\left(q_{T}, u\right), q_{2}\right)\right],\left[\left(\delta_{1}\left(q_{1}, \diamond\right), \delta_{T}\left(q_{T}, u\right), q_{h 2}\right)\right], \quad . \text { If } \mathrm{T} \text { is a row shuffle, then it is } } \\
& {\left[\left(q_{1}, \delta_{T}\left(q_{T}, u\right), \delta_{2}\left(q_{2}, a\right)\right)\right],\left[\left(q_{1}, \delta_{T}\left(q_{T}, u\right), \delta_{2}\left(q_{2}, \diamond\right)\right)\right], } \\
& {\left.\left[\left(q_{h 1}, \delta_{T}\left(q_{T}, u\right), \delta_{2}\left(q_{2}, a\right)\right)\right],\left[\left(q_{h 1}, \delta_{T}\left(q_{T}, u\right), \delta_{2}\left(q_{2}, \diamond\right)\right)\right]\right\} }
\end{aligned}
$$

defined as

$$
\begin{aligned}
\delta\left(\left(q_{1}, q_{T}, q_{2}\right), a\right)=\{ & {\left[\left(\delta_{1}\left(q_{1}, a\right), \delta_{T}\left(q_{T}, l\right), q_{2}\right)\right],\left[\left(\delta_{1}\left(q_{1}, \diamond\right), \delta_{T}\left(q_{T}, l\right), q_{2}\right)\right], } \\
& {\left[\left(\delta_{1}\left(q_{1}, \diamond\right), \delta_{T}\left(q_{T}, l\right), q_{h_{2}}\right)\right],\left[\left(\delta_{1}\left(q_{1}, a\right), \delta_{T}\left(q_{T}, l\right), q_{h_{2}}\right)\right], } \\
& {\left[\left(q_{1}, \delta_{T}\left(q_{T}, d\right), \delta_{2}\left(q_{2}, a\right)\right)\right],\left[\left(q_{1}, \delta_{T}\left(q_{T}, d\right), \delta_{2}\left(q_{2}, \diamond\right)\right)\right], } \\
& {\left.\left[\left(q_{h 1}, \delta_{T}\left(q_{T}, d\right), \delta_{2}\left(q_{2}, a\right)\right)\right],\left[\left(q_{h 1}, \delta_{T}\left(q_{2}, d\right), \delta_{2}\left(q_{2}, \diamond\right)\right)\right]\right\} }
\end{aligned}
$$

where $\left\{q_{1}, q_{h 1}\right\} \in Q_{1 h},\left\{q_{2}, q_{h 2}\right\} \in Q_{2 h}, q_{T} \in Q_{T}$ and $\{r, u, l, d\} \in T, \delta^{\prime}$ is defined from $\bar{R}_{h} \times \Gamma$ into $\bar{R}_{h}$. Thus we have $L(\mathrm{~A})=L_{1} \amalg_{\mathrm{T}} L_{2}$ and $L_{1} \amalg_{\mathrm{T}} L_{2}$ is a regular partial matrix array language.

\section{Theorem. 2}

For any two recognizable partial array languages $L_{1}, L_{2}, L_{1} \amalg_{\mathrm{T}} L_{2}$ is a recognizable partial array language, where $T \subseteq\{r, u\}^{*} \cup\{l, d\}^{*}$ is regular.

Proof. . et $L_{1}$ and $L_{2}$ be the two recognizable partial array languages accepted by OTPA, $\mathrm{A}_{1}$ and $\mathrm{A}_{2}$ are such that $L\left(\mathrm{~A}_{i}\right)=L_{i}, i=1,2$.

Define $\mathrm{A}_{i}=\left(\Sigma \cup\{\diamond\}, Q_{i}, Q_{h}^{i}, q_{0}^{i}, F_{i}, \delta_{i}\right)$, where $Q_{i}$ is set of states, $Q_{h}^{i}$ is the finite states associated with $\diamond, q_{o}^{i}$ is the initial state and $q_{0}^{i} \in Q_{i}$, for $i=1,2 . F_{i} \subseteq Q_{i} \cup Q_{h}^{i}$ are all final states. The transition function $\delta_{i}$ is defined as $\delta_{i}: Q_{p} \times Q_{p} \times(\Sigma \cup\{\vartheta\}) \rightarrow Q_{p}$.

The transition in $\mathrm{A}_{T}$ is as follows: a change from $\mathrm{A}_{1}$ to $\mathrm{A}_{2}\left(\mathrm{~A}_{2}\right.$ to $\left.\mathrm{A}_{1}\right)$ is taken as $u$ or $d(r$ or $l$, respectively. The input $\mathrm{P}$ is accepted by $\mathrm{A}$ iff each of $\mathrm{A}_{i}, i=1,2$ and $\mathrm{A}_{T}$ accepts $L_{1}$ and $L_{2}$ and $\mathrm{T}$ respectively.

Define $Q=Q_{1} \times Q_{T} \times Q_{2}, \quad Q_{0}=\left\{q_{0}^{1}, q_{T}, q_{o}^{2}\right\}, \quad$ where $\quad Q_{1}=Q_{1} \cup Q_{h}^{1} \quad$ and $\quad Q_{2}=Q_{2} \cup Q_{h}^{2} \quad, \quad$ thus $Q=\left(Q_{1} \cup Q_{h}^{1}\right) \times Q_{1} \times\left(Q_{2} \cup Q_{h}^{2}\right), F=F_{1} \times F_{T} \times F_{2}$.

The transition $\delta$ is defined as $\delta: Q \times Q \times(\Sigma \cup\{\oslash\}) \rightarrow 2^{Q}$ and is given as follows: 
$\delta\left[\left(q_{1}^{\prime}, q_{T}, q_{1}^{2}\right),\left(q_{2}^{1}, q_{T}, q_{2}^{2}\right), b\right]$

$=\left\{\left[\delta_{1}\left(q_{1}^{1}, q_{2}^{1}, b\right), \delta_{T}\left(\left(q_{T}, r\right), q_{1}^{2}\right)\right],\left[q_{1}^{1}, \delta_{T}\left(q_{T}, u\right), \delta_{2}\left(q_{1}^{2}, q_{2}^{2}, b\right)\right]\right.$,

$\left.\left[\delta_{1}\left(q_{1}^{1}, q_{2}^{1}, b\right), \delta_{T}\left(q_{T}, l\right), q_{1}^{2}\right],\left[q_{1}^{1}, \delta_{T}\left(q_{T}, d\right), \delta_{2}\left(q_{1}^{2}, q_{2}^{2}, b\right)\right]\right\}$

and

$\delta\left[\left(q_{1}^{1}, q_{T}, q_{1}^{2}\right),\left(q_{2}^{1}, q_{T}, q_{2}^{2}\right), \diamond\right]$

$=\left\{\left[\delta_{1}\left(q_{1}^{1}, q_{2}^{1}, \diamond\right), \delta_{T}\left(\left(q_{T}, r\right), q_{1}^{2}\right)\right],\left[q_{1}^{1}, \delta_{T}\left(q_{T}, u\right), \delta_{2}\left(q_{1}^{2}, q_{2}^{2}, \diamond\right)\right]\right.$,

$\left.\left[\delta_{1}\left(q_{1}^{1}, q_{2}^{2}, \diamond\right), \delta_{T}\left(q_{T}, l\right), q_{1}^{2}\right],\left[q_{1}^{1}, \delta_{T}\left(q_{T}, d\right), \delta_{2}\left(q_{1}^{2}, q_{2}^{2}, \diamond\right)\right]\right\}$

where $q_{1}^{1}, q_{2}^{1} \in Q_{1}, q_{T} \in Q_{T}, q_{1}^{2}, q_{2}^{2} \in Q_{2}, b \in \Sigma$. By the above transition it can be easily verified that $L(\mathrm{~A})=L_{1} \amalg_{\mathrm{T}} L_{2}$ and hence $L_{1} \amalg_{\mathrm{T}} L_{2}$ is also recognizable.

\section{Conclusion}

The impact of shuffle on trajectories over a regular matrix partial array languages and on the recognizable partial array languages are studied. The procedure can be applied in automation and in recognition of certain classes of designs. This can be applied in understanding the transformation of shapes in Architectural application and in evolution of two dimensional patterns.

\section{References}

[1] J. Berstel and L. Boassan, Partial words and a theorem of Fine and wilf, Theoretical Computer Science, 218 (1999), 135-141.

[2] H. Geetha, D.G. Thomas, T. Kalyani and A.S. Prasanna Venkatesan, Shuffle on trajectories over finite array languages, in J.K. Aggarwal et.al (eds), International Conference on Combinatorial Image Analysis, Lecture Notes in Computer Science 6636, 2011, 261-274.

[3] H.Geetha, K.Sasikala, P.Venkatesh Babu and T.Kalyani Shuffle on trajectories Over Partial Array Languages presented in ICMTA 2020 and published in AIP Conference proceedings,volume2277,06November 2020 030001-1 - 030001-8.

[4] P. Helen Chandra, C.Martin-Vide, K.G. Subramanian, D.L. Van and P.S.P.Wang, parallel contextual array grammars and trajectories in Handbook of pattern recognition and computer vision.

[5] A. Mateescu, G. Rozenberg and A. Salomaa, Shuffle on trajectories: syntactic constraints, Theoretical Computer Science, 197 (1998), 1-56.

[6] D.K. Sheena Christy, V. Masilamani, D.G. Thomas, Atulya K. Nayar and Robinson Thamburaj, Shuffle on array languages generated by array grammars Mathematics for application, math.Appl. 3(2014), 17-31.

[7] F. Sweety, D.G. Thomas, V.R. Dare and T. Kalyani, Recognizability of partial array languages, The Journal of combinatorial Mathematics and Computational Computing (2007), 237-249. 